\title{
La gestione per processi della calcolosi renale
}

\author{
G. Quintaliani' ${ }^{1}$ F. Gori ${ }^{2}$, M. Lombardi ${ }^{3}$
}

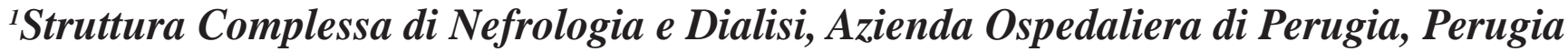 ${ }^{2}$ Sevizio Qualità ASL 1 Umbria, ${ }^{3}$ Unità Operativa di Nefrologia e Dialisi, Ospedale "S.M. Annunziata", Azienda 10, Firenze}

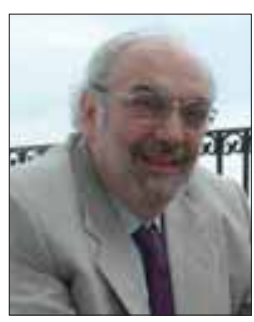

G. Quintaliania base per il sistema di gestione della qualità.

Il processo non è qualcosa di tecnico e freddo, fatto di procedure, attrezzature, flussi di attività, tecniche. Il processo ha "vita" in quanto è gestito da persone, ed ha origine, nella sua globalità da un mix di persone e metodologie, in cui le attrezzature sono solo uno strumento al servizio delle persone.

L'applicazione di tale approccio all'interno di una organizzazione sanitaria complessa, consente l'individuazione e quindi la necessità di governo, di processi gestionali e di processi sanitari.

Tale principio favorisce la visione globale all'organizzazione aziendale, rappresentandola attraverso un insieme di processi tra loro interconnessi (manageriali, clinici, in- fermieristici, amministrativi) in cui il paziente/ utente è coinvolto.

Infatti è bene evidenziare che ciò che il paziente/utente percepisce ha origine direttamente dai processi e solo indirettamente dalle singole funzioni.

Tradizionalmente le gestioni e i miglioramenti sono stati affrontati per funzioni (il responsabile, il coordinatore, l'aiuto, lo staff, gli orari, le guardie ecc) ma è a tutti noto che il valore si genera attraverso i processi, e non mediante le funzioni. In altre parole non è la patente che fa un campione, ma la bravura (Valentino Rossi docet).

Ragionare pertanto nella logica dei processi significa innanzitutto comprendere che, ai fini dell'efficacia e del miglioramento, non ha senso ottimizzare le funzioni aziendali in quanto tali. Una analisi delle norme ISO 9000: 2000, consente di leggere i principi di Gestione della Qualità come un "sistema" ispirato a una strategia di tipo unitario, di cui l'approccio per processi rappresenta la chiave interpretativa.

Uno dei passaggi chiave nella gestione per processi è l'identificazione e la delimitazione dei singoli pro- cessi che compongono l'attività sanitaria nel suo insieme.

La tipologia e il numero appropriato dei processi individuabili dipende dalle scelte aziendali che tendono a far prevalere la gestione delle interdipendenze tra $\mathrm{i}$ processi, oppure la garanzia di un maggior controllo sul raggio d'azione processuale.

Esistono due possibili approcci, non alternativi, ma complementari:

1) la mappatura delle attività a partire dalla struttura organizzativa, con cui vengono individuate le attività all'interno di ciascuna funzione rispetto a un output definito dalla struttura organizzativa stessa, per chiarire ciò che effettivamente viene svolto, indipendentemente dai mansionari degli operatori;

2) la definizione dei processi a partire dagli obiettivi definiti in base alle esigenze del paziente. La mappatura dei processi avviene sulla base delle aspettative del paziente che la direzione ritiene di dover soddisfare e di quelli che l'azienda considera $\mathrm{i}$ propri fattori critici di successo.

In questo contesto il processo assi- 


\section{Giornale di Tecniche \\ Nefrologiche \\ \& Dialitiche \\ Anno XVI n ${ }^{\circ} 3$ \\ (C) Wichtig Editore 2004}

stenziale, costituisce un insieme di attività strutturate e misurate, progettato per produrre un output specifico per il paziente, ma non solo, anche per i servizi che concorrono alla sua realizzazione.

Pertanto un processo può comprendere una serie di attività che si svolgono contemporaneamente o in successione all'interno di più funzioni aziendali. Da qui l'esigenza di una visione integrata e interfunzionale delle attività gestionali.

\section{La litiasi renale}

Si stima che a metà degli anni novanta, in Italia, fossero oltre un milione gli individui affetti da nefrolitiasi (17 pazienti ogni 1.000 abitanti ) e con necessità di ricorrere alle prestazioni del SSN (Statistiche della Sanità. Roma ISTAT 1992 e 1995).

Necessità che sembra essere progressivamente aumentata nel tempo: si è passati da 60,000 ricoveri nel 1988 ad oltre 80,000 nel 1993 (1), per poi arrivare a circa 104,000 ricoveri ordinari e, 32,000 DH nel 2002 nonostante una maggiore attenzione e filtro sui ricoveri impropri. Si deve notare che ben il $14 \%$ di questi pazienti ha dovuto ricorrere a procedimenti chirurgici o a un numero impressionante (circa 50,000/anno) di sedute di litotripsia extracorporea (ESWL), per una spesa annua di circa 180 milioni di euro (2). Per affrontare l'evento litiasi renale con la gestione per processi, dobbiamo distinguere due momenti fondamentali:

1) la diagnosi e il trattamento in elezione;

2) la colica renale e il follow-up.

\section{La diagnosi e il trattamento in elezione}

Ovviamente sembrano due momenti che si fondono insieme, tuttavia hanno dei significati assolutamente diversi e devono usufruire di percorsi e strutture differenziate.

L'istituzione di un ambulatorio della calcolosi infatti, lungi dall'essere un atto formale è la base di una serie di interconnessioni aziendali che devono essere attentamente vagliate.

Generalmente si parte scegliendo un medico che si è occupato del problema con più o meno competenze professionali e si richiede l'ufficializzazione dell'apertura dell' ambulatorio. E quanto di più sbagliato si possa fare. Sarà necessario invece che si metta il paziente e non la funzione al punto centrale della attività.

Prima dell'apertura dell' ambulatorio infatti andranno valutate con attenzione le ricadute che questa nuova attività avrà almeno su due componenti fondamentali: i servizi esterni (ecografia, TAC, laboratorio ecc), l'attività interna del reparto (quanto lavoro viene sottratto al reparto, se abbiamo risorse sufficienti per aggiungere anche questa attività a quelle ordinarie, se siamo ben consapevoli che se POI andremo a chiedere più risorse umane la risposta potrebbe essere negativa). Inoltre deve essere attentamente considerato che mai una attività qualunque essa sia, può essere svolta da un solo sanitario. La scelta di dedicare un solo sanitario a qualsivoglia attività è un errore che rende debole anche il suo lavoro perché fa intendere scarsa integrazione e flessibilità.

Una volta stabiliti bene i carichi di lavoro e gli impegni dovremo fare un notevole sforzo per collegarci ai servizi e stabilire con loro dei protocolli operativi (ad esempio, come riservare alcuni accessi per l'ecografia, il laboratorio e quant'altro). Dovremo fare uno sforzo notevole per predire le quantità in modo da non lasciare spazi liberi o ritrovarci con notevoli liste di attesa. La lista di attesa di una attività nuova è indice negativo che evidenzia scarsa attenzione alla programmazione. È bene ponderare con attenzione i carichi di lavoro.

Il paziente deve essere ben informato. È necessario che venga adeguatamente istruito sia verbalmente che con opuscoli su alcuni fatti importanti. Una delle componenti più neglette è l'istruzione sul come si eseguono gli esami (tipicamente vescica piena per l'ecografia), sulle ore degli appuntamenti, sugli orari delle visite di fol- low-up che devono essere effettuate in ambiente tranquillo, possibilmente al pomeriggio (non dimentichiamo che tipicamente i pazienti sono giovani e quindi con una attività lavorativa e che se lo svolgimento delle indagini ha richiesto alcune mattine almeno la visita sia loro favorevole). Inoltre va spiegato il fatto che la litiasi è una malattia subdola per cui si deve informare il paziente che non è saggio scomparire quando la sintomatologia si attenua o scompare; a questo proposito dovremo fare in modo di richiamare periodicamente $\mathrm{i}$ pazienti "persi" ed anche questo presuppone una buona organizzazione e la disponibilità di infermieri dedicati o amministrativi disponibili.

L'informazione al paziente di tutte le problematiche è fondamentale. Tra le richieste arrivate al sito www.renalgate.it la più frequente riguarda la calcolosi. Le richieste spaziano da quali esami fare, quale dieta seguire, dove rivolgersi ecc. È chiaro che un sito internet non può dare spiegazioni a tutto anche se una buona parte delle risposte più frequenti possono essere fornite: http://www.renalgate.it/lombardi_file/FAQ\%20SULLA \%20NEFROLITIASI1.htm, tuttavia è necessario che non solo all'interno dei singoli ambulatori si abbia materiale per l'informazione ma che la presenza di un ambulatorio di calcolosi sia adeguatamente rappresentata nella carta dei servizi aziendali, nel sito web aziendale o ricorrendo alla ospitalità su siti specifici di nefrologia. Alla fine di questo veloce excursus una raccomandazione che vale per tutti i tipi di paziente ma giova ripeterla. La documentazione ambulatoriale dovrebbe essere sempre disponibile (ovviamente in osservanza alla legge sulla privacy), perché il paziente potrebbe andare incontro a una colica e sapere se e dove è posizionato un calcolo o gli eventuali fattori di rischio potrebbe essere molto utile; insomma non si pretende che le cartelle siano lasciate sul tavolo, ma nemmeno dentro un classificatore chiuso a chiave nello studio del "responsabile" dell'ambulatorio che guarda caso è in ferie!! Insomma l'organizzazione di un am- 
bulatorio di litiasi, come altri ambulatori, è una attività che dovrebbe essere gestita come vari processi che si intersecano e che si dividono per poi ricongiungersi nelle linee finali per assistere il paziente nel miglior modo.

\section{La colica renale}

Ancora più importante è aver stabilito come ci si comporta con una colica renale che arriva dal Pronto Soccorso (PS). È una evenienza comune che va anticipata e quantificata bene.

Per quantificare il problema sarebbe buona prassi attingere ai database del PS in cui sono ben descritti i casi di colica renale arrivati e l'esito che hanno avuto: quanti sono stati ricoverati, dove, le eventuali complicanze ecc.

Attingendo per esempio ai dati della Azienda Ospedaliera di Perugia si è potuto appurare che ben il $68 \%$ dei casi di colica renale che si presentano al Pronto Soccorso viene rinviato a casa con terapia medica antalgica. Solo il $32 \%$ dei casi viene ricoverato, ma ancora una volta l'astenteria del PS si sobbarca il lavoro maggiore con un $18 \%$ dei ricoveri. In totale quindi il PS sbriga ben 1'86\% dei problemi legati alla calcolosi che si presentano per ricovero (3).

È ovvio che instaurare dei buoni rapporti con il PS è fondamentale. La consulenza nefrologica della colica renale lungi dall'essere un aggravio di lavoro, si dimostra un eccellente punto di intercettazione del paziente e delle sue problematiche. Anche in questo caso la disponibilità di materiale illustrativo e di numeri di telefoni da contattare per iniziare un percorso adeguato di diagnostica o di follow-up è assolutamente indispensabile.

Una volta stabilito il numero si dovrebbe valutare l'incidenza di ricovero e la durata (ancora i data base aziendali sono essenziali con le diagnosi ICD9). Anche in questo caso il paziente dovrebbe essere al cen- tro del processo e quindi si dovrebbe aver stabilito bene il processo dell' accoglienza di una colica renale. Di base è la presenza di linee guida (LG) particolareggiate stilate insieme al PS, alla radiologia, urologia ecc, dove sia ben scritto quale deve essere il primo livello di indagine o d'intervento. Lo stilare linee guida solo per il reparto in oggetto, per quanto corrette e specialistiche, rende le LG assolutamente prive di una qualche rilevanza aziendale che diventa invece pregnante quando le LG siano state condivise da più reparti insieme che condividono percorsi e processi diversi, ma comuni in molti tratti.

Sarà necessario quindi aver stabilito per iscritto un qualche schema di trattamento antalgico (uno degli strumenti migliori per evitare un ricovero non sempre effettivamente necessario) e poter poi rimandare a casa il paziente. Infatti solo dopo aver ben istruito il paziente sulla terapia antalgica sarà possibile convincerlo che la classica "flebo" ospedaliera non è miracolosa! Il dotare il paziente di una schema antalgico scritto fa sì che anche la guardia medica o il medico di famiglia siano più tranquilli nel trattare un paziente a casa di cui si sia accertata in modo ragionevole la diagnosi e che non sia riportato al PS a causa di manifestazioni legate al dolore, magari accompagnato da uno stuolo di parenti eccessivamente preoccupati se non preda di manifestazioni di dissenso manifesto.

Altro momento importante è il follow-up. Se il paziente torna a casa dovremo aver ben stabilito come e dove rivederlo e quali indagini debbano essere fatte. È invalso l'uso da parte del medico di famiglia di riempire richieste urgenti per superare la lista di attesa. E del tutto ovvio che in caso di colica renale la richiesta di urgenza è l'unica arma per poter effettuare velocemente esami strumentali. Ma gestire un'attività di nefrolitiasi comporta saper programmare e quindi prevedere, seppur in maniera approssimativa, il numero di coliche che po- tranno richiedere l'assistenza in un dato periodo di tempo e quindi saper garantire un numero di accessi liberi e pronti per le necessità imposte da quest'attività garantendo l'urgenza. Sembra molto difficile ma in realtà non lo è se cerchiamo di venire incontro alle esigenze del paziente e se siamo in grado di fare programmazione e organizzazione. Programmazione e organizzazione che spesso pretendiamo da altri (gli staff aziendali) ma che spesso siamo proprio noi clinici a snobbare con il risultato di complicare inutilmente le cose. Ricordiamoci inoltre che è classica una delle espressioni più frequenti che si sentono nei servizi diagnostici: «Possibile che sia sempre tutto urgente?». Anche gli altri colleghi hanno il nostro stesso diritto di lavorare con un minimo di programmazione, facciamo in modo che quando possibile tutto quello che facciamo sia organizzato.

Il ricorso poi alla logica PDCA (Plan Do Check, Act) che poi in fondo significa vedi se hai fatto bene e casomai modifica quello che non va, può essere effettuata solo se avremo ben chiaro quello che facciamo. Se invece ogni volta dobbiamo partire da capo e se veramente riteniamo che ogni caso è un caso a sé, vuol dire che saremo anche degli ottimi clinici, ma che non possiamo fregiarci del ruolo così ambito di "responsabile".

\section{Conclusioni}

La gestione per processi ci insegna a suddividere la nostra attività in piccoli percorsi in modo da poter controllare le strozzature, i punti critici e i punti di forza.

Questo sforzo ancor di più, deve essere messo in atto nel caso della litiasi renale che coinvolge così tante professionalità dentro le aziende sanitarie e che rappresenta un grande investimento in salute per i pazienti.

Sarebbe addirittura auspicabile poter introdurre nell'ambito organizzativo il Responsabile del processo 


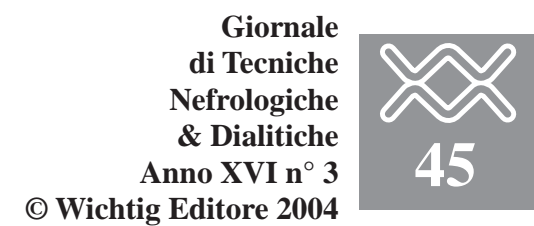

assistenziale, figura che rende possibile la gestione efficace del percorso complessivamente, diventando il baricentro delle relazioni fra il paziente e i vari professionisti che intervengono in esso. Tale posizione consente di mediare gli interessi, talvolta non concordi, dei vari soggetti. Egli deve essere in grado di monitorare efficacemente tutte le attività che costituiscono il processo eliminando il più possibile le difficoltà di comunicazione interne al processo e con i soggetti esterni. Il responsabile del processo dovrebbe avere l'autorità a promuovere le scelte che possano influenzare efficacemente il percorso assistenziale.

\section{BIBLIOGRAFIA}

1. Serio A, Fraioli A. Epidemiology of Nephrolithiasis. Nephron 1999; 81 (suppl.1): 26-30.

2. Tuccillo S, Giannattasio P, Ciuffi M, et al. Prevenzione della nefrolitiasi calcica primitiva. Giorn It Nefrol 2000; 4: 339-47.

3. Taddei M, Quintaliani G, Lombardi M, Tazza D. I DRG nella calcolosi urinaria. Atti del $4^{\circ}$ Simposio Chiancianese sulla Calcolosi Renale. 2002; 10: 113-24. 